\title{
16
}

\section{Software Quality Classification Model based on McCabe's Complexity Measure}

\author{
Ryouei Takahashi \\ NTT Information and Communication Systems Laboratories \\ Yokosuka - shi, Kanagawa, 238 - 03, Japan \\ $(T E L)+81-468-59-8312(F A X)+81-468-59-3726$
}

\begin{abstract}
A software quality classification model based on McCabe's complexity measure is in vestigated. It is experimentally shown using regression and discriminant analyses that program fault density (number of faults per 1000 source lines of code) depends on the complexity of the functional unit rather than on that of each of the modules. To identify the best model, stepwise selection method improved by AIC (Akaike Information Crite rion) is applied to regression and discriminant analyses. A functional unit is the parti tioning unit of the sof tware function and consists of several modules that are combined with data that is commonly referred to or updated. This partitioning is done in the ear ly phases of the software life cycle and experiments show that interfaced complexity among functional units is associated with software faults. The concept of an "extented cyclomatic number $\left(S_{1}{ }^{\prime}\right)$ " - an extension of McCabe's design complexity measure $S_{1}$ from an inner - functional - unit control structure to a between - functional - unit control structure - is proposed taking this complexity tendency into consideration and is exper imentally studied. It is well known that $S_{1}$ is equal to the number of predicates +1 . In the metric $\mathrm{S}_{1}{ }^{\prime}$, the nesting depth of predicate nodes for invoking module path selection is taken into consideration. The number of predicates whose scope is confined to a functional unit can be separately counted from the predicates whose scope extends to differ ent functional units. This software quality classification model with the extended cy clomatic number enables project managers to control sof tware quality.
\end{abstract}

\section{Keywords}

Software quality classification, McCabe's complexity measure, regression analysis, discriminant analysis, AIC

\section{Introduction}

The relationship between software quality and complexity metrics has been researched for many years [Li, Cheung, 1987]. These experiments have shown that com plexity metrics are interrelated with each other and several complexity metrics are related to sof tware quality to some degree. Taking the relationship between complexity metrics and software quality into consideration, various software quality prediction models have been developed [Takahashi, Wakayama, 1994; Munson, Khoshgoftaar, 
1992; Rodriguez, Tsai, 1987].

Complexity metrics, such as the cyclomatic number for measuring the complexity of the software control structure [McCabe, 1976], are used as indicators for program modularization, revising specifications, and test coverage. In addition, they have been used in software quality prediction models, whose purposes include predicting fault numbers through multivariate regression analysis and identification of error-prone modules based on discriminant analysis. Those models, however, are based on metric data and quality data contained in the program modules themselves and do not sufficiently take the functional relations between modules into consideration. Here, a mod ule is defined as the lowest level unit of hierarchical functional partitioning [De Marco, 1986] and corresponds to a function in C-coding [Richie, Kernighan, 1978], and module's size was experientially shown to be of the order of $10^{1} \sim 10^{2}$ SLOC (source lines of code).

In our experiments, on the contrary, the relationship between complexity metrics and sof tware quality were investigated using multivariate regression analysis. The results show that program fault density (fault number per 1000 SLOC), which we use as a measure of software quality, does not depend on the complexity density (ex. cyclomatic number per 1000 SLOC) of every fault module but on the complexity of the functional unit with modules functionally related to the fault module. In other words, variance analysis shows that program fault density is mostly characterized by functional units. A functional unit is a program unit partitioned in the early phases of software devel opment from the standpoints of data structure or process and corresponds to a certain hierarchical level of partitioning sof tware. Each unit consists of several modules (coincident with functions in $\mathrm{C}$ - coding) and functional unit's size is experientially shown to be of the order of $10^{3} \mathrm{SLOC}$, which is suitable for a programmer to design, to code and to test. Since a software quality control unit should be a manageable comprehensible evaluation unit, it generally accords with a functional unit.

The next phase of our investigation was characterization of functional units with complexity metrics, using the techniques of discriminant analysis with stepwise selection method improved by AIC (Akaike Information Criterion) estimates [Takahashi, Wakayama, 1994; Akaike, 1974], but unfortunately the ratio of correct classification ratio was not very high. In the work reported here, we used 12 complexity metrics ob tained from the viewpoints of source lines of code [Yu, Smith, Huang, 1991], the cyclomatic number that measures the number of predicates [McCabe, 1976], the fan - in and fan - out that measures the complexity of information flow [Henry, Kafura, 1981], the number of external variables [Halstead, 1977], and comment lines [Woodfield, Dunsmore, Shen, 1981]. The main reason the missclassifination ratio was high in the previous work is probably that these 12 complexity metrics could not sufficiently represent the functional relationship among the modules that compose a functional unit. Agresti has measured the interfaced complexity among modules by the the "context - coupling" metric [Agresti, Evanco, 1992] and "relative complexity" metric [Card, Agresti, 1988]. The former metric counts the number of data commonly used among modules to measure the interconnection of modules and the latter metric counts the number of invoking modules and $\mathrm{I} / \mathrm{O}$ variables. But data obtained by using these metrics represent interfaced complexity from every module's viewpoint and can't sufficiently represent the complexity of a functional unit. On the other hand, McCabe proposed integration com plexity measure $S_{1}$ [McCabe, Butler, 1989] to measure complexity of an inner - func- 
tional - unit control structure in the design phase and to count the number of predicates that select the invoking module path. But our experiments using regression analysis have shown that the correlation coefficient between $S_{1}$ and program fault density is not so high $\left(\mathrm{R}^{2}=0.10\right)$ and only the interfaced complexity among modules in a functional unit is not thorough enough to represent the complexity tendency of a functional unit.

To solve this problem, the "extended cyclomatic number" $\mathrm{S}_{1}$ ' is proposed, considering the relationship among functional units, and the validation of this metric is empirically and statistically studied. The proposed cyclomatic number extends the concepts of in tegration complexity measure $S_{1}$. The applied area of McCabe's complexity metric is extended from an inner-functional - unit control structure to a between -functional unit control structure. A functional unit control structure is represented by a gragh. In the graph, $\mathrm{S}_{1}$ is defined as number of edges - number of nodes +2 , where nodes corre spond to invoking functions or invoking predicates and edges correspond to executing sequences of nodes. It is well known that $S_{1}$ is equal to the number of predicates +1 . In the metric $\mathrm{S}_{1}$ ', nesting depth of predicate nodes for invoking module path selection is taken into consideration. In it, the number of predicates whose scope is confined to a functional unit can be separately counted from those predicates whose scope extends to different functional units. Consequently, the interfaced complexity among different functional units can be obtained using the metric $S_{1}$ ' and the accuracy of software qual ity evaluation model can be increasingly improved. In fact, our experiments on regres sion analysis applied to a small scale program (about 10 thousand of SLOC) have shown that the correlation coefficient between $S_{1}$ ' and the program fault density is very high $\left(\mathrm{R}^{2}=0.70\right)$. This sof tware quality classification model with the extended cyclomat ic number that shows the interfaced complexity among functional units enables project managers to control sof tware quality.

\section{The extended cyclomatic number}

\subsection{The applied area of the metric $S_{1}$,}

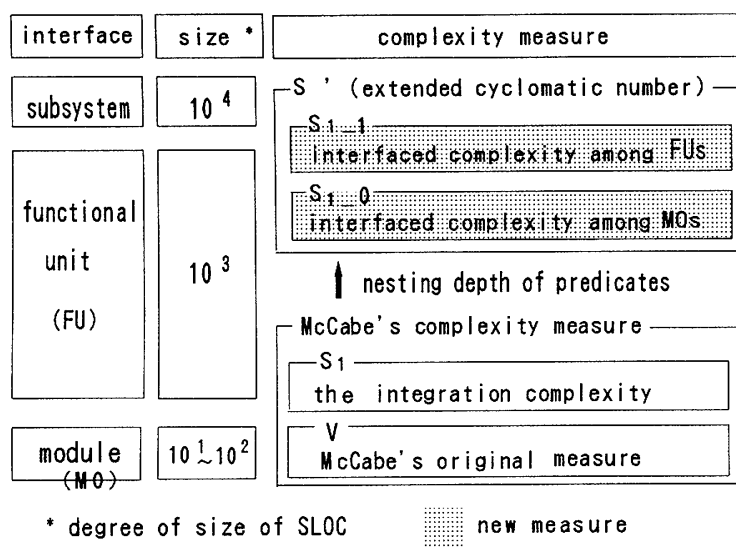

Fig. 1. Background
The applied area of the ex tended cyclomatic number $\mathrm{S}_{1}{ }^{\prime}$ is shown in Fig. 1. $\mathrm{S}_{1}{ }^{\prime}$ is expressed as the thin hatched areas. To measure interfaced complexity among software quality evaluation units of a certain hierarchical level par titioning, complexity metrics are divided into three classes: an inner-module level complexity metric (to measure complexities of an inner module whose size is of the order of $10^{1} \sim 10^{2}$ SLOC and McCabes's original complexi - 
ity metric is part of this class), an inner - functional - unit level complexity metric (to measure complexities of an inner functional unit that has the size of the order of $10^{3}$ SLOC, where a functional unit consists of several modules and McCabe's design com plexity measure $S_{1}$ belongs to this class), and an inner - subsystem level complexity metric (to measure complexities of an inner subsystem that has the size of the order of $10^{4}$ SLOC, where a subsystem consists of several functional units). The metric $\mathrm{S}_{1}$ ' is an inner-subsystem level metric that measures interfaced complexity among functional units. In $\mathrm{S}_{1}$ ', nesting depth of predicate nodes for invoking module path selection is taken into consideration. This metric extends the concepts of integration complexity measure $S_{1}$ proposed by McCabe. The purpose of $S_{1}$ is to measure the complexity of an inner functional unit control structure and it is well known that it is equal to the number of predicates +1 . With $S_{1}$, inner logic of every module is not taken into consid eration; a path that does not influence the interrelationship between modules is elimi nated. The metric $S_{1}$ itself is an extended metric of McCabe's original cyclomatic number $\mathrm{V}$ from an inner-module-control structure to a between-module-control structure. In this reseach, the applied area of McCabe's complexity metric is extended from an inner - functional - unit control structure to a between - functional - unit control structure. In the metric $\mathrm{S}_{1}$ ', the number of predicates whose scope is confined to a functional unit can be separately counted from those predicates whose scope extends to dif ferent functional units. A software control unit corresponds to a software functional unit, and a software functional unit corresponds to a subtree of a hierarchical module control structure [De Marco,1986]. We call this tree call \& called module tree. The par titioning function correponds to a call \& called module tree decomposed into several subtrees.

\subsection{Concepts of the metric $S_{1}$,}

\section{(1) Re - definition of integration complexity metric $S_{1}(G)$}

Before we describe the concepts of $\mathrm{S}_{1}$ ', the concepts of McCabe's design complexity measure $S_{1}$ are reviewed. The metric $S_{1}$ is an extension of McCabe's original complexity metric from an inner-module control structure to a between-module (an in ner - functional - unit) control structure. A module is assumed to correspond to a function in $\mathrm{C}$ coding. The metric $\mathrm{S}_{1}(\mathrm{G})$ measures the complexity of a functional unit graph $\mathrm{G}$. A node in $\mathrm{G}$ not only corresponds to a function in $\mathrm{C}$-coding but also to a predicate for invoking function path selection. An edge in $\mathrm{G}$ corresponds to an invoking function or invoking function path selection. $S_{1}(G)$ does not reflect the inner logic complexity of every function. Hence, $S_{1}(G)$ is defined as

$$
\mathrm{S}_{1}(\mathrm{G})=\mathrm{e}_{1}-\mathrm{n}_{1}+2 \text {, }
$$

where $e_{1}$ is the number of edges and $n_{1}$ is the number of nodes in graph $G$. On the oth er hand, the purpose of the original McCabe's complexity metric [McCabe, 1976] V(F) is to measure the complexity of the inner - logic of every function $F$, and $V(F)$ is defined as $\mathrm{e}-\mathrm{n}+2$, where $\mathrm{e}$ is the numer of edges and $\mathrm{n}$ is the number of nodes in graph $\mathrm{F}$. In graph $\mathrm{F}$, a node corresponds to an instruction statement or a decision statement in $\mathrm{C}$ coding, and an edge signifies the execution of sequences of nodes. It is well known that $\mathrm{V}(\mathrm{F})$ is equal to the number of predicates plus one in structured programming, and is equal to the number of regrions (Euler's formula in a connected plane graph). The 


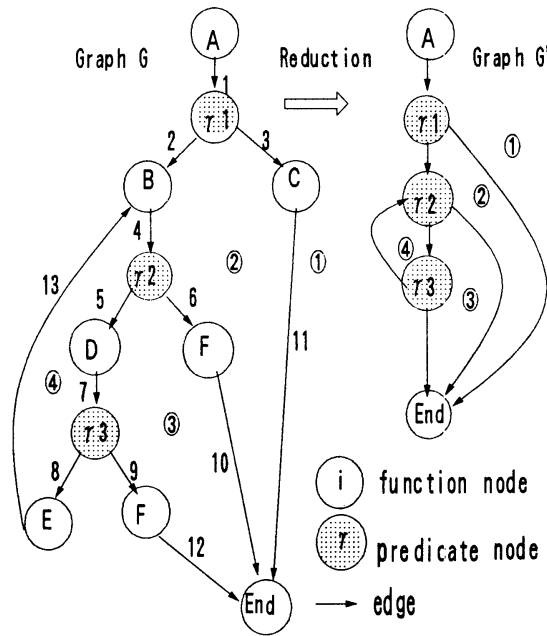

Fi g. 2. NCC abe' $s$ in tegration me asure $S_{1}(G)$.

graph theory is also applicapable to a between-module control structure $S_{1}$, so the metric $S_{1}$ is considered to be an extention of McCabe's original complexity metric $\mathrm{V}$ and it is proved that $S_{1}(G)$ equals the number of predicates (plus 1 ) for invoking function path selection.

\section{[An application of $S_{1}$ ]}

An example of $S_{1}(G)$ is illustrated in Fig. 2. This graph consists of six functions denoted A, B, C, D, E, F, an end node, and 3 predicates, $\gamma 1, \gamma 2$, and $\gamma 3$. A invokes $\mathrm{C}$ when $\gamma 1$ is satisfied, and invokes B when $\gamma 1$ is not sat isfied. B invokes $F$ when $\gamma 2$ is satisfied, and invokes $\mathrm{D}$ when $\gamma 2$ is not satisfied. D invokes $\mathrm{F}$ when $\gamma 3$ is satisfied, and invokes $\mathrm{E}$ when $\gamma$ 3 is not satisfied. $\mathrm{E}$ invokes $\mathrm{B}$ recursively. Then $S_{1}(G)=$ number of edges - number of nodes $+2=13-11+2=4$ and is equal to the number of predicates $(3)+1$. Further more, as you can see in the graph, $S_{1}(G)$ is equal to the number of regions (1) (4)). You can also see that $S_{1}$ in a reduced graph $G^{\prime}$ is consistent with $S_{1}$ in the original graph $\mathrm{G}$, where a reduced graph $\mathrm{G}^{\prime}$ includes predicate nodes but not function nodes.

\section{(2) Defnition of the extended cyclomatic number $S_{1}$ '}

The extended cyclomatic number $\mathrm{S}_{1}$ ' extends the concepts of integration complexity measure $S_{1}$, considering the effects of interfaced complexity among functional units. The metric $S_{1}{ }^{\prime}(G)$ classifies predicate nodes into $S_{1}-0(G)$ and $S_{1} \ldots 1(G)$, taking the nesting depth (i.e. scope) of predicate nodes for invoking module path selection into consideration [Howatt, Baker, 1989]. The number of predicates whose scope is confined to a functional unit can be separately counted from those predicates whose scope ex tends to different functional units. The scope of predicate node $\mathrm{X}$ includes a set of functions whose executions are controlled by decision statement $X$, where nesting of predi cate nodes is taken into consideration. When a predicate node $\mathrm{Y}$ is directly or indirectly controlled by predicate node $\mathrm{X}$, not only functions whose executions are directly controlled by predicate $\mathrm{X}$, but also functions whose executions are controlled by predicate $\mathrm{Y}$ are included in the scope of $\mathrm{X}$.

\section{[Defnition of $\left.S_{1}^{\prime}\right]$}

The metric $\mathrm{S}_{1}$ ' is divided into two metrics.

(A) $S_{1} \_0(G)$ : The extended cyclomatic number of inner quality control unit subtrees • .. This class consists of predicates in $G$ whose scope is confined to functions defined in a functional unit $G . S_{1} \quad 0(G)$ is the number of predicates (plus +1 ) belonging to this class.

(B) $S_{1} \quad 1(G)$ : The extended cyclomatic number among different quality control unit subtrees ... This class consists of two sets of predicates. The first set consists of predi- 
cates in $G$ whose scope in cludes functions defined in another functional unit $G^{\prime} . S_{1_{2}} 0: 4$ The second set consists of $S_{1} 1: 2$ predicates in another functional unit G' whose scope in cludes functions defined in $G$ itsef. $S_{1} \quad 1(G)$ is the sum mation of the number of pred icates that belong to the first set and the number of predi cates that belong to the second set.

$\mathrm{S}_{1} \quad 0(\mathrm{G})$ measures the complexity of an inner - functional - unit control structure and $\mathrm{S}_{1}-1(\mathrm{G})$ measures the complexity of a between functional-unit (i.e. an inner - subsystem - unit) control structure.

\section{[An application of $S_{1}$ ']}

An example of $\mathrm{S}_{1}{ }^{\prime}\left(\mathrm{S}_{1} \ldots, \mathrm{S}_{1}\right.$

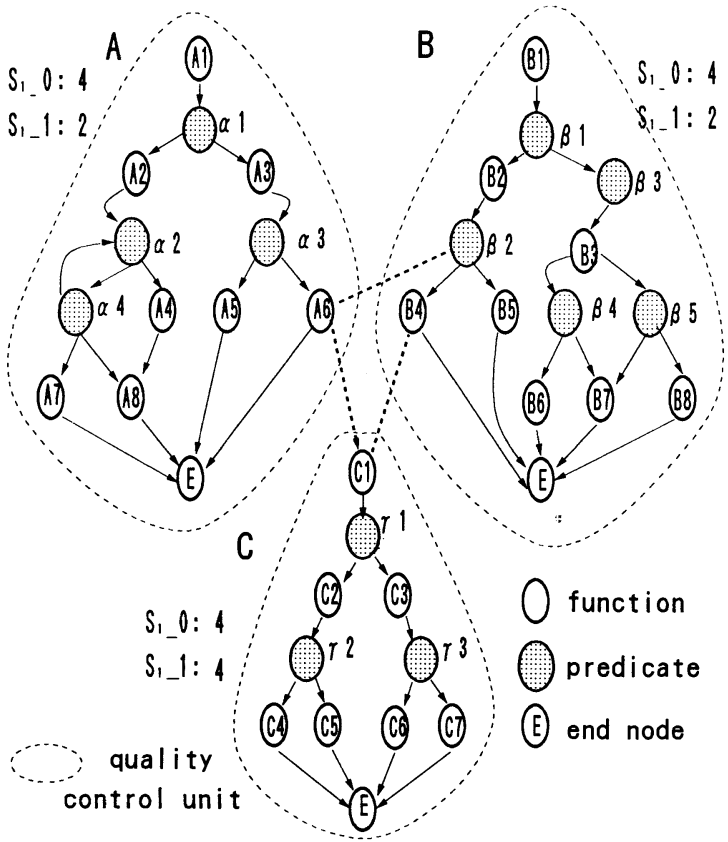

Fig. 3. An application of $S_{1}$ '.

1 ) is shown in Fig. 3, where

the graph indicates information flow among functions including predicates for invok ing function path selection, and $\rightarrow$ denotes the invoking function or invoking predicate in an inner - quality - control subtree, and $\cdots>$ denotes the invoking function among dif ferent quality control - unit subtrees. This example has three software quality control units, A, B, and C. In the graph the end node of every quality control unit is also shown. Subtree $\mathrm{A}$ is connected with subtree $\mathrm{C}$ by $\operatorname{arc} \mathrm{A} 6->\mathrm{C} 1$, where $\mathrm{A} 6$ and $\mathrm{C} 1$ are functions. Subtree $\mathrm{B}$ is connected with subtree $\mathrm{A}$ by $\operatorname{arc} \beta 2->\mathrm{A} 6$ and is also connected with sub tree $\mathrm{C}$ by arc $\mathrm{B} 4->\mathrm{C} 1$, where $\beta 2$ is an executing condition (predicate) of function $\mathrm{A} 6$ and B4 is a function. From subtree C's standpoint, as the scope of three predicates $\gamma 1$, $\gamma 2, \gamma 3$ includes only functions $\mathrm{C} 2, \mathrm{C} 3, \cdots \mathrm{C} 7$ defined in $\mathrm{C}$ itself, it follows that $\mathrm{S}_{1}$ $0(\mathrm{C})=3+1=4$. In addition, as the execution of function $\mathrm{C} 1$ is controlled by predicates $\alpha \overline{1}$, $\alpha 3, \beta 1$, and $\beta 2$ in functional units $\mathrm{A}$, and $\mathrm{B}$, it follows that $\mathrm{S}_{1}, 1(\mathrm{C})=4$. Similarly from subtree A's standpoint, predicates $\alpha 2$ and $\alpha 4$ ( $\alpha 4$ has three branches so the number of predicates of $\alpha 4$ is 2) only control execution of functions A4, A7, and A8 defined in A itself, so it follows that $S_{1} \quad 0(A)=3+1=4$. Since the scope of predicates $\alpha 1, \alpha 3$ not only includes functions defined in $\mathrm{A}$ itself but also includes function $\mathrm{C} 1$ defined in functional unit $\mathrm{C}$, it follows that $\mathrm{S}_{1} \ldots 1(\mathrm{~A})=2$. Similarly, $\mathrm{S}_{1} \_0(\mathrm{~B}), \mathrm{S}_{1} \_1(\mathrm{~B})$ are counted. The calculation is summarized as follows:

$$
\begin{aligned}
& \text { - } \mathrm{S}_{1} \_(\mathrm{A})=4=\left\{\alpha 2, \alpha 4^{*} \vdots+1, \mathrm{~S}_{1} \_1(\mathrm{~A})=2=\{\alpha 1, \alpha 3\}\right. \\
& \text { - } \mathrm{S}_{1} \_(B)=4=\{\beta 3, \beta 4, \beta 5\}+1, \mathrm{~S}_{1} 1(\mathrm{~B})=2=\{\beta 1, \beta 2\} \\
& \text { - } \mathrm{S}_{1} \_0(\mathrm{C})=4=\{\gamma 1, \gamma 2, \gamma 3\}+1, \mathrm{~S}_{1} \_1(\mathrm{C})=4=\{\alpha 1, \alpha 3, \beta 1, \beta 2\} \text {. }
\end{aligned}
$$

Here \{\} is a set of predicates, $\alpha \overline{4}^{*}$ has 3 branches and is counted as two 
predicates, $S_{1} \_0(X)=$ number of elements of set $X$ plus one, and $S_{1} \_1(X)=$ number of elements of a set $\mathrm{X}$.

\section{A case study}

\subsection{Sample spaces}

Metric and quality data were obtained from the DBMS (Database Management Sys tem) maintenance program developed by NTT Information and Communication Sys tems Laboratories. This program is a subsystem of the DBMS. Our program is written in $\mathrm{C}$ and consists of $10 \mathrm{KDSI}$ (thousands of delivered source instructions). Its function is to change $\mathrm{DB}$ attributes and directories during online operations. It consists of seven excluded software quality control units. Each quality control unit consists of several routines, and a routine coincides with a compliled file designated by *.c in UNIX. Each file consists of several functions in $\mathrm{C}$ coding. Overall, the DB maintenance program consists of 50 routines, and 144 functions. The sample spaces of the seven units are scribed as follows: the 1st group (the initializer/terminator is designated by in Fig ures 4,5 , and 7 ) and consists of 7 routines, 21 functions, 1.4 KDSI (shortened to ( , 7,21,1.4) hereafter); the 2nd group (the SQL analyzer) consists of $(\bigcirc, 9,39,2.8)$; the 3rd group (the garbage collector), $(\times, 7,3,0.5)$; the 4 th (the data transporter) of $(\triangle, 42,10,2.4)$; the 5 th (the SQL parameter generator), $(\square, 19,8,1.6)$; the 6 th (the SG parameter reformalizer), ( $\boldsymbol{\square}, 6,6,0.6)$; and the 7 th (common modules), $(\boldsymbol{\Lambda}, 10,7,0.9)$.

\subsection{Complexity metrics}

In this investigation, we used 12 complexity metrics obtained from the viewpoints of source lines of code [Yu, Smith, Huang, 1991], the cyclomatic number that counts the number of predicates [McCabe, 1976], the fan - in and fan - out that measures the com plexity of information flow [Henry, Kafura, 1981], the number of external and static variables [Halstead, 1977], and comment lines [Woodfield, Dunsmore, Shen, 1981]. A summary is given in Table 1.

Table 1. Summary of complexity metrics used in the case study

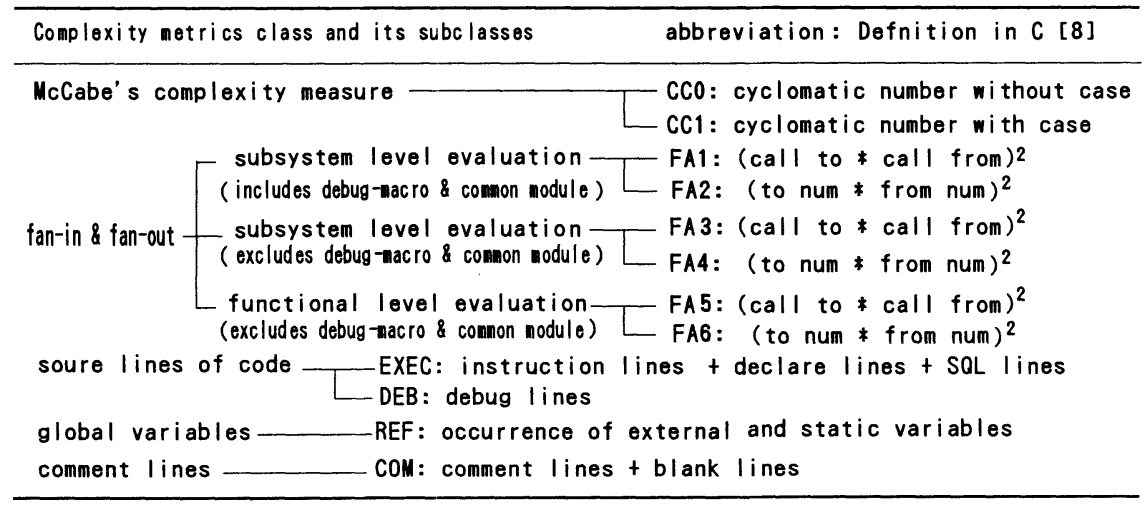




\section{[The metric "fan-in \& fan-out"]}

The metric "fan-in \& fan -out" investigated in this study is not consistent with what Henry \& Kafura originally proposed. In this paper, "fan - in \& fan-out" is confined to the local flow [Henry, Kafura, 1981] of function invoking, and is classified into 6 pat terns by the combination of three conditions: whether common modules or debug macros are excluded or not in every quality control unit, whether invoking functions among different functional units are taken into consideration or not, and whether total occurrences of invoking functions are taken into consideration or not. In the table,

- call to $=$ number of distinct functions that call to other functions

- call from = number of distinct functions that are called from other functions

- to num = total numbers of function calling

- from num = total numbers of function called

\subsection{Relationship between program fault density and module complexi - ty density}

The sof tware control units were the 50 routines mentioned in section 3.1. Experiments show that correlation coefficients between fault number and software complexity met rics are high, and the complexity metrics are interrelated [Takahashi, Wakayama, 1994]. Faults are detected and corrected after the implementation phase of the software cycle. However, a relationship between program fault density (fault number per 1000 source lines of code) and program module complexity density was not found. As a typi cal example, the cyclomatic number without case $(\mathrm{CC} 0)$ per 1000 source lines of code versus program fault density is shown in Fig. 4. The determinant coefficients $R^{2}$ of the model was low $(=0.07)$.

\subsection{Analysis of program fault density variance from the program func - tion viewpoint}

To identify the most suitable regression model, stepwise selection method [SPSS, 1991] improved by AIC (Akaike Information Criterion) [Akaike, 1974] was applied to

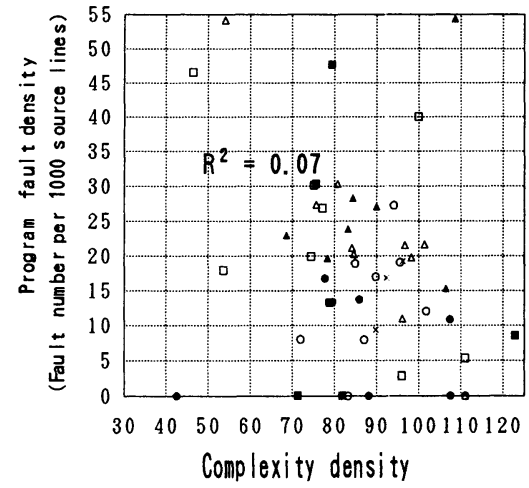

Fig. 4. Corplexity density versus progra fault density.

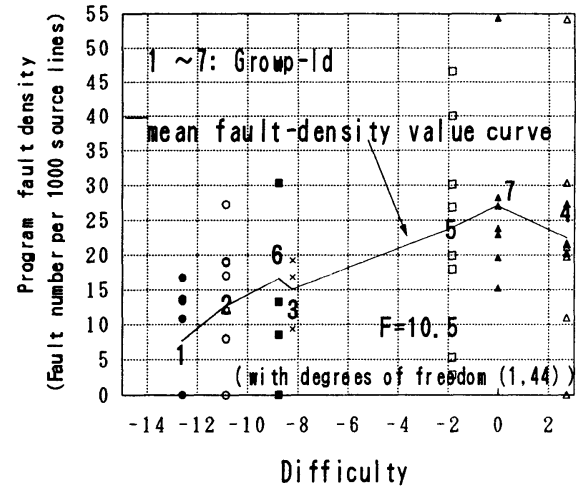

Fig. 5. Analysis of fault density variance. 
select the main complexity - metric factors (the procedure to select complexity-metric factors is based on AIC estimates and details are explained in section 3.5, because the idea is the same between regression analysis and discriminant analysis). The AIC procedures are based on the maximum likelihood estimation method and the least number of complexity metrics. The results of the minimum AIC estimated model were as fol lows: $\mathrm{R}^{2}$ was 0.44 . The $\mathrm{F}$ - test value was 10.52 for the "software function", 7.04 for the "distinct functions number density", 6.28 for the "comment lines (COM) density", 3.12 for the "global variables (REF) density", and 2.56 for the "cyclomatic number with case's (CC1) density". The item "software function" has the highest F-test value, in dicating it is the primary factor affecting program density. These results mean we need a complexity metric that can distinguish software functional units. The results of regression analysis is illustrated in Fig. 5. The y-axis is for program fault density. The $\mathbf{x}$-axis represents software quality control unit "difficulty", whose values are item scores obtained from the minimum AIC regression model. The item score of "software function" agrees with a partial regression coefficient. If the item score increases, program fault density tends to increase, which is why we call this item "difficulty". Each sample item score is equal if the sample belongs to the same functional unit. The set $(1,2, \cdots, 7)$ in the Fig. 5 signifies the functional units, and each functional unit's mean fault density value curve is also presented.

\subsection{Discriminant analysis to classify software functional units by complexity metrics}

The most suitable software quality classification model can be obtained by the step wise selection method [SPSS, 1991] with AIC estimates [Akaike, 1974]. This method, which takes the number of complexity metrics into consideration [Takahashi \& Wakayama, 1994], is thought to be an improvement on the Wilks $\Lambda$ criteria [Rao, 1973]. The relationship between the AIC estimate and Wilks $\Lambda$ is discussed in detail in [Takahashi, Wakayama, 1994]. The procedure begins with the selection of the metric with which the lowest AIC estimated discriminant model M1 can be constructed. Then, the second metric is selected among the residual metrics, and by adding it to M1, the low est AIC estimated model M2 can be obtained. The AIC estimate of M2 is smaller than that of M1. This procedure of lacating the next added metric continues until metrics can no longer be added or until the adding of any residual metric to the last model AIC estimate no longer gets smaller. The most suitable discriminant model obtained in this study was with an F-test value of 7.42 , an AIC estimate of 364 , and a ratio of correct classification of $0.16=(23 / 144)$, where $23 \mathrm{~F}$ - test (AIC estimate) trials were performed with the stepwise selection method, and the last selected complexity metric was "fan - in \& fan - out (FA1)". The complexity metrics are interrelated (multicollineali ty), so we could not establish the unique contribution of every metric to the discriminant model. As a consequence, only the "fan - in \& fan-out number FA1" was selected among the 12 complexity metrics. The main reason the missclassification ratio was high is probably that these 12 complexity metrics can not sufficiently represent the functional relationship among the modules that compose a functional unit. In an at tempt to solve this problem, we applied our extended cyclomatic number and the validi ty of the metric was experimertally verified as described in section 3.6. The result shown in this section, i.e., that functional units can be classified by the metric "fan - in - 


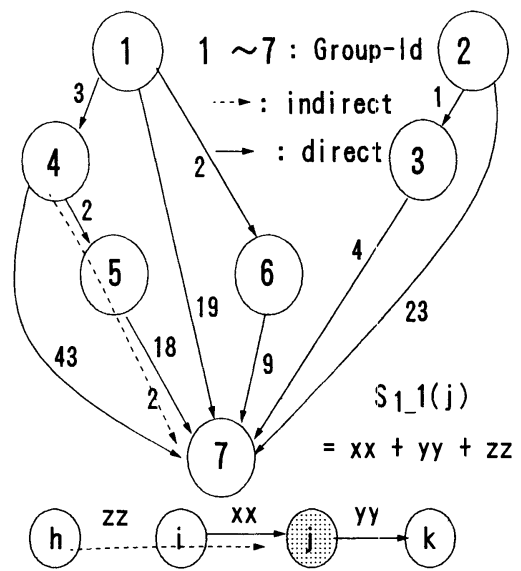

Fig. $6 . S_{1} 1$ in the case study.

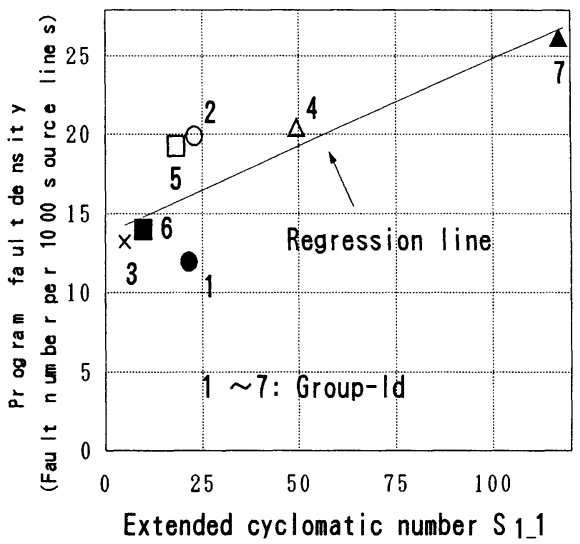

Fig. 7. $S_{1-1}$ versus program fault density

$\&$ fan-out" that measures the interfaced complexity among modules is consistent to the result represented in section 3.6, i.e., that program fault density has a high correla tion with extended cyclomatic number $S_{1} \ldots 1$ that measures the interfaced complexity among functional units, although the classification ratio of the most suitable discrimi nant model is not very high and the evaluation level of software quality is different be tween the model in this section and that in section 3.6.

\subsection{Extended cyclomatic number $S_{1}$ ' and program fault density}

The correlation between McCabe's integration complexity metric $S_{1}$ and program fault density (fault number per 1000 SLOC, where fault number is the summation of each module's fault number and SLOC is the summation of each module's SLOC) is not very high ( $R^{2}$ of the model was 0.10 ). We assume that program fault density mainly stems from interfaced complexity among functional units. The measurement of interfaced complexity among seven software quality control units is summarized in Fig. 6 , where Os represent quality control units (QUs), and values on the arrows denote the degree of the effects of the predicates (i.e. number of predicates) in a certain QU whose scope occupies functions defined in another QU. In this sample, not only predicates that give effects directly (denoted by $\rightarrow$, which indicates interfaced complexity between two QUs) but also predicates that give effects indirectly (denoted by $-->$, which indi cates interfaced complexity among more than two QUs) are taken into consideration. $\mathrm{S}_{1} \ldots 1(\mathrm{X})$ is the summation of values on input arrows to a certain quality control unit $\mathrm{X}$ and values on output arrows from $X$ and can be thought as an indicator of the total in terfaced complexity of X. Through Fig. 6, it has been seen that group-id 7 (a group is a functional unit) has the highest interfaced complexity among the seven functional units. The relationship between program fault density and the metric $S_{1} \ldots 1$ is shown in Fig. 7. The determinant coefficient $R^{2}$ between program fault density and $S_{1} \ldots 1$ was very high $\left(R^{2}=0.70\right)$, indicating that the more accurate software quality classification model could be obtained by taking nesting of predicates nodes into consideration. But, the correlation coefficient between program fault density and $\mathrm{S}_{1}{ }_{-} 0$ was low - 
$\left(R^{2}=0.0006\right)$ in this case study.

\section{Conclusion}

The extended cyclomatic metric $\mathrm{S}_{1}$ ' for measuring the interfaced complexity among functional units has been proposed for the purpose of evaluating software quality (program fault density) and its validity has been experimentally verified. $\mathrm{S}_{1}$ ' is an exten tion of McCabe's integration metric $S_{1}$ from an inner - functional control structure to a between-functional control structure, taking the scope (nesting depth) of predicate nodes among functional units into consideration. The metric $\mathrm{S}_{1}$ ' was applied to a DBMS maintenance program developed by NTT whose volume is about 10 thousand source lines of code (KSLOC) written in C language. The results of our experiments are summerized as follows:

(1) The correlation coefficient between program fault density and cyclomatic complexi ty density is not very high, where the software evaluation unit is a module, (i.e., the function or routine with the size of the order of $10^{1} \sim 10^{2}$ SLOC) which is the lowest level of hierarchical partitioning of software functions.

(2) It has been shown using the minimum AIC estimate regression model that the "software function" is the main factor that causes program faults, where "software function" is classified into several categories coincident to the above functional units. A functional unit consists of several interrelated modules and has the size of the order of $10^{3}$ SLOC and is a certain hierarchical - level partitioning unit of the sof tware function.

(3) Even with the minimum AIC estimate discriminant model, the "software function" could not be characterized by complexity metrics that measure module - level complexi ty.

(4) Although classification ratio of the most suitable discriminant model is not very high, the metric "fan-in \& fan-out", which measures the interfaced complexity among modules, is the most effective factor for the discrimination of the "software function".

(5) The correlation between McCabe's integration complexity metric $S_{1}$ and program fault density is not very high.

(6) The determinant coefficient $\mathrm{R}^{2}$ between program fault density and our extended cy clomatic number $S_{1} \ldots 1$ was very high $\left(R^{2}=0.70\right)$, indicating that a more accurate soft ware quality classification model can be obtained by taking the scope of predicates nodes among functional units into consideration.

To construct a more accurate software quality classification model, it is necesarry to extend sample spaces in order to confirm model validity, to investigate the conditional variable complexity (i.e., these variables are used for invoking function paths [McClure, 1978]), and to evaluate stepwise selection method to reach the minimum AIC es timate discriminant model. All of these remain for future work.

\section{References}

[1] Agresti, W. W. and Evanco, W. M. (1992) Projecting software defects from analyz- 
ing Ada designs," IEEE Trans. Software Eng., 18, 11, 988 - 97.

[2] Akaike, H. (1974) A new look at the statistical identification: IEEE Trans. Automatic Conrol, AC-19, 6, 716-23.

[3] Card, D. N. and Agresti, W. W. (1988) Measuring software design complexity: The Journals of Systems and Software, 8, 185 - 97.

[4] De Marco, T. (1986) Structured analysis and system specification (translated into Japanese by Takanashi,T. and Kuroda,J., and published by Nikkei BP Inc.).

[5] Halstead, M. H. (1977) Elements of Software Science: Elsevier North - Holland,Inc.

[6] Henry, S. and Kafura, D. (1981) Software strucreture metrics based on information flow: IEEE Trans. Software Eng.,SE - 7,5, 510 - 18.

[7] Howatt, J. W. and Baker, A. L. (1989) Rigorous defnition and analysis of program complexity measures: An example using nesting: The Journal of Systems and Soft ware, $10,139-50$.

[8] Li, H. F. and Cheung, W. K. (1987) An empirical study of software metrics: IEEE Trans. Sottware Eng., SE -13, 6, $697-708$.

[9] McCabe, T. J. (1976) A complexity measure: IEEE Trans. Software Eng.,SE - 2, 4, $308-20$.

[10] McCabe, T. J. and Butler, C. W. (1989) Design complexity measurement and Testing: Communications of AC.M, 32, 12, 1415-25.

[11] McClure,C.L.(1978) A model for complexity alnalysis: 3th ICSE, 149-57.

[11] Munson, J. C. and Khoshgoftaar, T. M. (1992) The detection of fault-prone programs: IEEE Trans. Software. Eng., 18, 5, 423 - 33.

[12] Rao, C. R. (1973) Linear statistical inference and its applications 2nd edition: John Wiley \& Sons, $244-47$ and 556 - 7.

[13] Richie, D. M., Johnson, S. C., Lesk, M. E. and Kernighan, B. W. (1978) The C programming language: The Bell System Technical Journal, 57, 6, 1991 - 2019.

[14] Rodriguez, V. and Tsai, W. T. (1987) A tool for discriminant analysis and classi fication of software metrics: Information and Sof tware technogology, 29, 3, 137 - 150

[15] SPSS Inc. (1991) SPSS Statistical Algorithms 2nd Edition: 76 - 80 and 230 - 32.

[16] Takahashi, R. and Wakayama, H. (1995) Discriminative efficiency methodology for validating software quality classification models: Systems and Computers in Japan, 26 , 5, 1-18.

[17] Woodfield, S. N., Dunsmore, H. E. and Shen, V. Y. (1981) The effects of modularization and comments on program comprehension: 5th ICSE, 215 - 23.

[18] Yu, W. D., and Smith, J. P. and Huang, S. T. (1991) Software Productivity measurements: COMPSAC'91, 558-64.

\section{Ryouei Takahashi}

received his B.S. and M.S. degrees in 1974 and 1976, respectively, from Waseda Univer sity, and then joined NTT. He has been engaged in DIPS real-time software development of maintenance systems, and software quality and productivity evaluation sys tem. His research interests are in software complexity metrics, quality, and statistical modeling. Currently, he is a senior research engineer at NTT Information and Com munication Systems Laboratories. He is a member of the I.E.I.C.E and Information Processing Society of Japan. 